\section{GRP-155 RECONCILIATION ERRORS ASSOCIATED WITH ANTIRETROVIRAL TREATMENT}

doi:10.1136/ejhpharm-2013-000276.155

JA Dominguez Menendez, B Balzola Regout, A Llona Armada, FJ Goikolea Ugarte, LM Mendarte Barrenechea, MJ Yurrebaso Ibarreche. Basurto Hospital, Pharmacy, Bilbao, Spain

Background Electronic health records systems facilitate reconciliation of patients' medicines. However, chronic medicines prescribed by hospital physicians and dispensed only at hospitals such as HIV treatments, are not yet recorded in primary care records and sometimes the dose and frequency are not correctly recorded in patients' medical histories when they enter hospital.

Purpose To describe and analyse the discrepancies in HIV chronic treatments prescribed by hospital practitioners at admission to hospital.

Materials and Methods From June to October 2012, data of patients admitted with antiretroviral medicines were collected. HIV patients admitted to the Infectious Diseases Service or treated chronically in other hospitals were excluded. The pharmacist compared the computerised prescriptions at admission with the current HIV treatment recorded in the pharmacy chronic prescriptions dispensed programme (Farhos). In the event of discrepancies the pharmacist informed the physician/nurse and corrected the order. Non-justified discrepancies were notified and classified as reconciliation errors.

Results 68 patients' treatments were analysed (Average age: 46 years. 44 men, 24 women). 49 patients were admitted to the emergency ward (E) and 19 to other wards (O). The average HIV drugs per patient were 2.2 . In 17 patients $(25 \%)$ the treatment was not correct $(22.5 \%$ of $E$ and $31.5 \%$ of $O)$.

23 discrepancies were found in 150 medicines ( 0.33 per patient). 12 of these were associated with darunavir $(41.6 \%$ of darunavir treatments were wrong). Classified by reconciliation errors: dose/ frequency incorrect (16), omission (5), wrong drug (2).

Conclusions Incorrect prescriptions at admission of chronic hospital medicines such as HIV treatments cause a great number of reconciliation errors. Complex regimes, such as those including darunavir, facilitate prescription errors. Until HIV medicines are recorded in patients' primary care records or recording is complete in hospital medical histories, the pharmacy data and pharmacist interventions are needed to guarantee the correct treatment. Due to the results, HIV stock drugs were removed from the Emergency Service.

No conflict of interest.

\section{GRP-156 RECONCILIATION ERRORS AT CARDIOLOGY UNIT ADMISSION}

doi:10.1136/ejhpharm-2013-000276.156

A Luna Higuera, R Asensi Diez, M Toca Muñoz, MA Rosado Souviron, IM Muñoz Castillo. HRU Carlos Haya, Pharmacy, Malaga, Spain

Background The reconciliation process detects medicines errors and is a key point for improving patient safety.

Purpose To analyse the incidence, type and severity of reconciliation errors at Cardiology Unit admission.

Materials and Methods Descriptive prospective observational study from October-November 2011 in patients admitted to the Cardiology Unit in a tertiary hospital. Demographic data studied: sex and age.

The patient's usual chronic treatment, obtained by comprehensive interview of the patient and by reviewing the clinical history, was compared with the medicines prescribed on admission in order to identify: no discrepancies (ND), intentional discrepancies (ID)
(Formulary substitutions/modifications in response to a patient's clinical status) and apparently unexplained discrepancies requiring clarification with the physician (DRCs). After clarification, Reconciliation Errors (REs) (discrepancies resulting in physician order changes) were classified by type and severity.

Results 113 patients were included. The median age was $71.2 \pm 10.4$ years. $56.2 \%$ were male. Only 50 patients were reconciled due to logistical reasons.

528 medicines investigated: 159 ND (30.11\%), 256 ID (48.49\%) and 113 DRCs $(21.40 \%)$.

After clarification, 47 (41.59\%) DRCs were REs, while 5 discrepancies $(4.42 \%)$ (2 patients) could not be resolved. $8.91 \%$ of prescriptions $(47 / 528)$ were REs.

REs affected 22 (45.83\%) of the 48 real study patients. The average number of REs per patient was $2.14 \pm 1.21$.

Types of RE were: omissions $(n=31)$, different dose/route/ frequency $(\mathrm{n}=7)$, unnecessary medicines $(\mathrm{n}=5)$, wrong medicine $(\mathrm{n}=3)$ and incomplete prescription $(\mathrm{n}=1)$.

In terms of severity, REs were distributed as follows: No error, but possible $(n=10)$, error that does not reach the patient $(n=25)$, error reaching but not harmful $(n=11)$ and error requiring monitoring $(\mathrm{n}=1)$.

Conclusions The process of taking a pharmacotherapeutic history at hospital admission is inadequate since almost half of the patients showed REs, mostly omissions.

Although most REs caused no harm, if perpetuated at discharge, they might have worse consequences and/or affect the effectiveness of treatment.

The pharmacist's work in hospitalisation units is vital to reduce errors in care transitions and represents an opportunity to develop integral pharmaceutical attention in order to increase patient safety.

No conflict of interest.

\section{GRP-157 REDUCING THE INCIDENCE OF "MISSED DOSES" AT NORTH BRISTOL NHS TRUST (NBT)}

doi:10.1136/ejhpharm-2013-000276.157

'J Smith, ' $\mathrm{J}$ Hamer, 'A Mundell, 'N Mogford, 'R Brown, ${ }^{2} \mathrm{~J}$ Gillard, ${ }^{3} \mathrm{~F}$ Hamill. 'North Bristol NHS Trust, Pharmacy, Bristol, UK; ${ }^{2}$ North Bristol NHS Trust, Cardiology, Bristol, UK; ${ }^{3}$ North Bristol NHS Trust, Clinical Audit \& Assurance, Bristol, UK

Background In 2010 the National Safety Agency published a report on reducing harm from omitted and delayed medicines in hospital: 'Missed Doses occur when a medicine is not given to a patient when prescribed and may result in harm'.

NBT invested in Patient Safety, including: the Safer Patients Initiative (SPI2) and the Southwest Quality and Patient Safety Improvement Programme (SWOPSI). There are various causes of missed doses, our initial focus was drug unavailability.

NBT was set targets by the local commissioning body of reducing missed doses by $20 \%$ by $2010 / 11$, and a further $15 \%$ by $2011 / 12$. Purpose To reduce the incidence of missed doses due to drug unavailability. The objectives were to: Raise awareness of the effects on patients; Understand the reasons for missed doses and to introduce an e-audit tool for ward use.

Materials and Methods Using improvement methodology, tests of change were trialled and spread to 40 wards:

Phase 1: February 2010-July 2010:

We determined the criteria for missed doses and developed an e-audit tool using Plan Do Study Act (PDSA) cycles.

Phase 2: August 2010-April 2011:

The Ward e-audit tool was tested then spread; Wards were given a stock medication location report and Pharmacy prioritised missed doses 